\title{
Role of surrounding gas in the outcome of droplet splashing
}

\author{
David A. Burzynski and Stephan E. Bansmer \\ Technische Universität Braunschweig, Institute of Fluid Mechanics, Hermann-Blenk-Strasse 37, \\ 38108 Braunschweig, Germany
}

(Received 7 December 2018; published 3 July 2019)

\begin{abstract}
This study investigates the influence of the surrounding gas on a droplet impacting a smooth dry glass surface at high Weber and Reynolds numbers. It was performed using a flywheel experiment and different gases at ambient pressure. We analyzed the splashing outcome by measuring the size, velocity, and angle of the secondary droplets and by calculating the total volume ejected. We show that gas entrapment is not the mechanism responsible for splashing at high Weber and Reynolds numbers. We demonstrate that splashing is influenced by the density, followed by the viscosity, and last by the mean free path of the surrounding gas. Furthermore, the surrounding gas primarily affects the number of secondary droplets ejected and their ejection angle, whereas the droplet size and horizontal velocity are independent of the surrounding gas properties. We provide the first theoretical expression for the total volume ejected using the theory of Riboux and Gordillo [Phys. Rev. Lett. 113, 024507 (2014)], which attributes the secondary droplet generation to a lift force experienced by spreading lamella. The relationship between the ejected volume and the splashing parameter is described by a power function.
\end{abstract}

DOI: 10.1103/PhysRevFluids.4.073601

\section{INTRODUCTION}

When a droplet impacts solid surfaces at high velocities, it atomizes into many secondary droplets (see Fig. 1). This splashing phenomenon is very common in nature and in many technical applications, such as coating, cooling, gas turbines, and aircraft icing [1-4]. Several investigations have been carried out to analyze the splashing threshold or the maximal spreading diameter. Nevertheless, almost nothing is known about the secondary droplets that are generated [1,5]. Theoretical and empirical models have been proposed to describe this phenomenon as a function of the impact conditions and the fluid and gas properties [6,7]; however, these theories have only been validated for impact velocities slightly higher than the splashing threshold. Thus, can these theories explain the physics behind the impact of high velocity droplets?

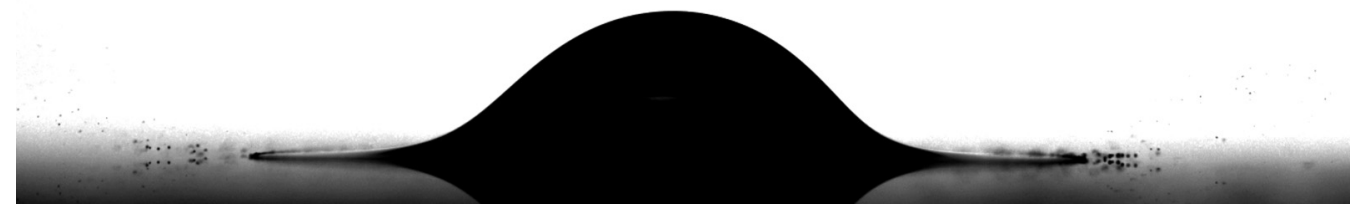

FIG. 1. A water droplet of diameter $3.7 \mathrm{~mm}$, traveling at $10 \mathrm{~m} / \mathrm{s}$, impacts a smooth dry glass surface surrounded by air. This example of a prompt splash shows the ejection of secondary droplets from the spreading rim at the surface; scale bar $500 \mu \mathrm{m}$. 
(a) frontal view

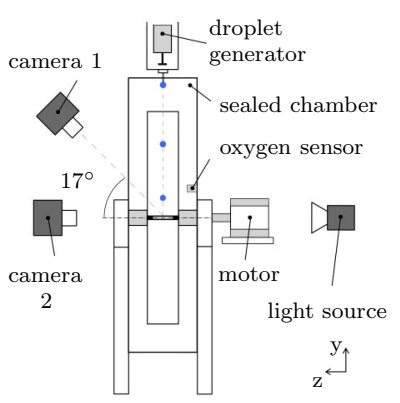

(b) lateral view

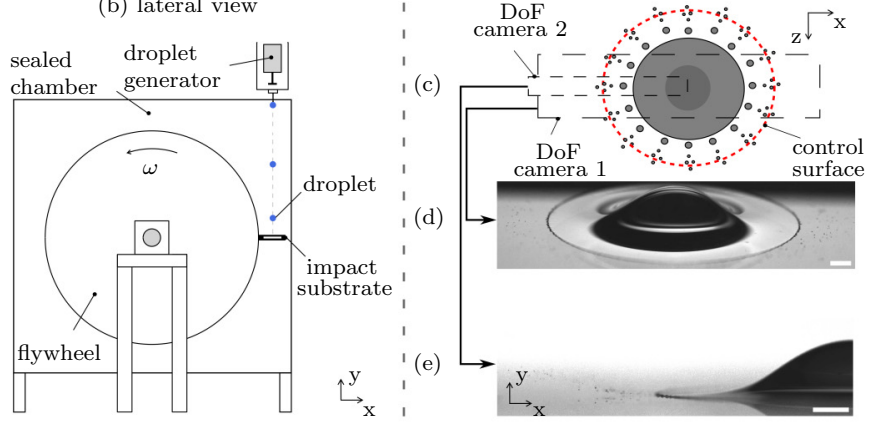

FIG. 2. Experimental method. (a) Sketch of the flywheel experiment showing the shadowgraph setup, which consists of two high-resolution cameras and one laser light source. (b) Sketch demonstrating the mode of operation and the substrate position where the impact takes place. (c) Schematic representation of a splashing droplet observed from above, showing the depth of field of each camera and the control surface used to calculate the total ejected volume. (d), (e) An example of a large water droplet $D \approx 3.7 \mathrm{~mm}$ impacting the substrate at $U \approx 10 \mathrm{~m} / \mathrm{s}$, recorded at the same instant; scale bars $1 \mathrm{~mm}$.

It has been demonstrated that the surrounding gas plays an important role during the impact process [8]. In the early stages of the impact, the droplet deforms just before the contact with the surface and entraps a small amount of gas [9], while during the spreading phase the gas determines the dynamics of the thin lamella [10]. Xu et al. [8] demonstrated that at reduced pressure splashing can be suppressed; however, it has been recently suggested that it is the gas density and not the pressure that affects the splashing behavior [11]. On the contrary, Jian et al. [12] have postulated that this process is dominated by the gas viscosity with a minor contribution from the gas density. Therefore, it is still unclear which property of gas affects splashing the most.

We performed a systematic experimental study using different gases at constant absolute pressure to understand how the surrounding gas affects the entrapped gas bubble and the generation of secondary droplets. We analyzed the morphology and size of the entrapped gas bubble and performed a full characterization of the splash first by measuring the size, velocity, and angle of the droplets and then by calculating the total number and volume ejected. We make use of the theories of Mandre et al. [13] and Hicks and Purvis [14] to analyze the gas entrapment phenomena at the early stage of impact. We combine the theory of Riboux and Gordillo [15] with our experimental data to study the mechanism responsible for the ejection of secondary droplets. We aim to provide a simplified formulation for the total volume ejected, which is needed for modeling in many technical applications.

\section{EXPERIMENTAL METHOD}

The experimental setup used in this study is shown in Fig. 2. Water droplets of diameter $D \approx$ $3.7 \mathrm{~mm}$ are formed and released by a droplet generator. These droplets fall freely due to gravity while a flywheel, on which the impact substrate is mounted, rotates at a constant angular velocity $\omega$. The relative velocity between a falling droplet and the impact substrate determines the impact velocity, which is held constant at $U \approx 10 \mathrm{~m} / \mathrm{s}$. The surface roughness of the glass substrate is $R_{a}=22 \mathrm{~nm}$. The properties of the water used are $\rho_{l}=998 \mathrm{~kg} / \mathrm{m}^{3}, \mu_{l}=1.003 \mathrm{mPas}$, and $\sigma_{l}=$ $72.75 \mathrm{mN} / \mathrm{m}$. This setup allows for experiments at $\mathrm{We}=\rho_{l} U^{2} D / \sigma_{l} \approx 4800$ and $\operatorname{Re}=\rho_{l} U D / \mu_{l} \approx$ 35000 , respectively. The entire flywheel is sealed within a chamber to allow the use of different gases. The oxygen concentration $\chi_{O_{2}}$ and the absolute pressure $p$ are measured next to the impact position to calculate the properties of the gas mixture $[16,17]$. Table I shows the calculated properties of the gases used in the experiment. The absolute pressure was constant for all experiments at $p_{\text {atm }} \approx 1$ bar. For each of the cases studied, more than 1000 impacts total have been evaluated at different elapsed times to provide a solid statistical database. 
TABLE I. Gas properties calculated during the experiments. The gas concentration $\chi$ is represented in parenthesis.

\begin{tabular}{|c|c|c|c|c|c|c|c|c|c|c|}
\hline & $\mathrm{He}(95 \%$ & $\mathrm{He}(70 \%$ & $\mathrm{H}_{3}(77 \%$ & $\mathrm{IH}_{3}(54 \%)$ & Air & )$_{2}(95 \%$ & $\operatorname{tr}(79 \%$ & $\mathrm{O}_{2}(41 \%$ & $\mathrm{O}_{2}(74 \%$ & $\mathrm{F}_{6}(39 \%)$ \\
\hline$\rho_{g}\left(\mathrm{~kg} / \mathrm{m}^{3}\right)$ & 0.22 & 0.48 & 0.83 & 0.94 & 1.21 & 1.32 & 1.56 & 1.46 & 1.67 & 3.22 \\
\hline$\mu_{g}(\mu \mathrm{Pas})$ & 20.3 & 18.9 & 12.7 & 14.8 & 18.2 & 20.3 & 21.7 & 16.1 & 15.2 & 16.4 \\
\hline$\lambda_{g}(\mathrm{~nm})$ & 173 & 110 & 55 & 60 & 66 & 70 & 69 & 53 & 47 & 36 \\
\hline
\end{tabular}

To visualize splashing, we used a shadowgraph technique with two high-resolution double-frame cameras $(4008 \times 2672$ pixel $)$ and a Nd:YAG laser with diffuser optics. A $180 \mathrm{~mm}$ lens with a teleconverter was connected to each camera, providing a maximal resolution of $5 \mu \mathrm{m} /$ pixel. Both cameras allow a global and a detailed view of the splash as shown in Figs. 2(d) and 2(e). To avoid false positive detection of the smallest droplets, for example due to pixel noise, we evaluated only the droplets with a minimum area of $3 \times 3$ pixels. This restriction permits the analysis of droplets larger than $15 \mu \mathrm{m}$. The use of conventional lenses with a limited depth of field (DOF) means that the droplets closest to the focal plane are likely to be detected, while the droplets further away are blurry or not detected at all (see sketch in Fig. 2). Additionally, larger droplets are more likely to be detected even if they are out of the focal plane; the detected droplet size is proportional to the DOF [18]. This proportionality biases the probability distribution function of the droplets, as the frequency of the smaller droplets tends to be underestimated. To correct for this, we calibrated the control volume in the depth direction by traversing a commercial target plate through the impact area. The calibration provides the correlation between DOF and droplet size, which is then used in the statistical results.

The synchronization of the cameras with the droplet generator and flywheel was performed using a delay generator. The delay generator received the trigger signal from a light barrier mounted on the flywheel and then sent two delayed signals: the first one to a solenoid, which smoothly hit the needle and released the droplet, and the second signal was sent some milliseconds later to the cameras that recorded the events.

\section{RESULTS AND DISCUSSIONS}

\section{A. The entrapped gas bubble}

We first analyzed the entrapped gas bubble, observed when the spreading film height is very thin, as shown in Fig. 3. The dynamics of this gas entrapment are described by the lubrication theory in the gas, which excludes any compressibility effect [19]; however, it has been pointed out that for high-velocity impacts this cannot be neglected [13]. According to Mandre et al. [13], the distance between the droplet bottom and the surface at which the droplet starts to deform is defined as $H=1.6 \epsilon^{1 / 3} D \mathrm{St}^{2 / 3}$, where $\epsilon=p_{\text {atm }}\left[D U^{7} \rho_{l}^{4} /\left(2 \mu_{g}\right)\right]^{-1 / 3}$ is the compressibility factor and $\mathrm{St}=2 \mu_{g} /\left(\rho_{l} U D\right)$ is the Stokes number. We used the properties of the gas mixtures in the experiments to calculate $H$ and obtained a variation between $100<H<150 \mathrm{~nm}$. One interesting observation is that in the experiments conducted with $\mathrm{He}(95 \%)$ the predicted height $H \approx 140 \mathrm{~nm}$ is smaller than the mean free path of gas molecules $\lambda_{g}=173 \mathrm{~nm}$, which implies that the continuum equations without slip correction are not able to properly describe the gas between the droplet and the surface. Hence, either lubrication theory with slip models or kinetic gas theory must be applied instead [20]. The model of Mandre et al. [13] suggests that at $H \leqslant \lambda_{g}$ the deformation of the droplet starts in a region where no gas molecule is probable to be found; therefore, gas entrapment should not be expected. Despite this, we observed that the gas is always entrapped. Based on their numerical simulations at reduced pressure, Mandre and Brenner [21] later suggested that in this particular case the gas beneath the droplet is compressed rather than drained out, which again leads to entrapment. To our knowledge, by changing the physical properties of the gas without reducing 

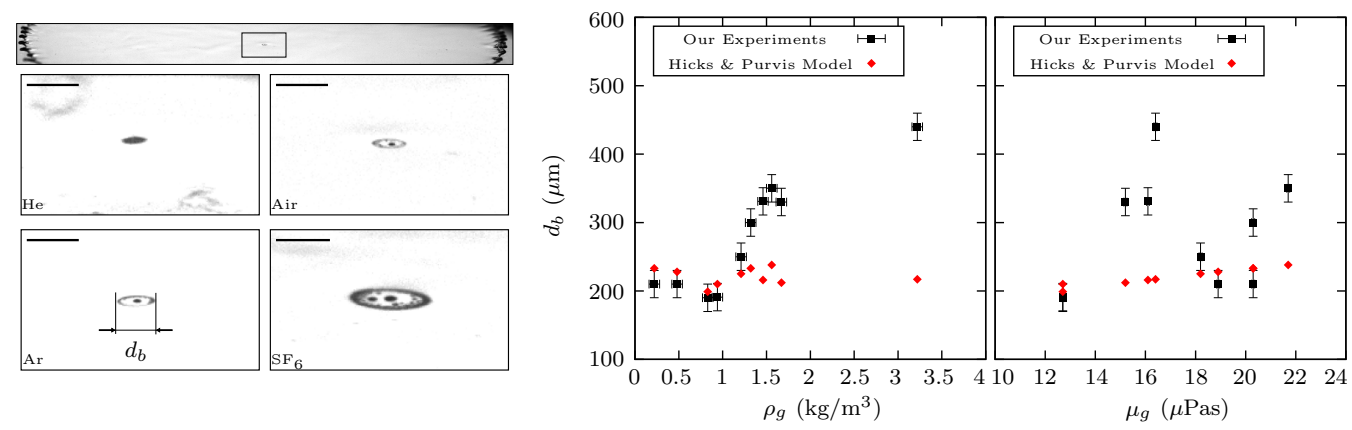

FIG. 3. Footprints of the gas entrapment mechanism. The top image on the left side shows the spreading rim and the entrapped gas within a box. The enlargements highlight the different morphologies that were observed; scale bars $500 \mu \mathrm{m}$. The diagrams show the diameter of the ring of microbubbles $d_{b}$ as a function of the gas density and viscosity.

the gas pressure, our study provides the first experimental evidence that supports this idea of Mandre and Brenner [21].

We also observed different morphologies for the different gases (see Fig. 3). Although the most common morphology observed was a ring of microbubbles with single or multiple larger bubbles in the center [22], a single bubble was observed only when using helium. Increased gas density resulted in more bubbles in the center and thicker rings. During the experiments with $\mathrm{SF}_{6}(39 \%)$, the primary droplet slightly deformed to an oblate before the impact. This was caused by the high stagnation pressure at the impact surface, which was generated by the flywheel rotation.

To conclude the analysis of the entrapped gas bubble, we measured the diameter of the ring of microbubbles $d_{b}$, which determines the position where the droplet contacts the substrate [22]. Although it has been suggested that either the density [11] or the viscosity [12] dominates the early stage of splashing, we found that the gas bubble diameter is strongly influenced by both $\rho_{g}$ and $\mu_{g}$ (see Fig. 3). To elucidate how each gas property affected the entrapment size, we compared the following two cases. In the first case, we picked out two gases with the same viscosity but significantly different densities, $\Delta \rho_{g} \approx 83 \%$ [He(95\%) and $\mathrm{O}_{2}(95 \%)$ ], and we observed that the diameter $d_{b}$ varied approximately $30 \%$. However, we selected another two gases with similar densities but different viscosities $\Delta \mu_{g} \approx 30 \%\left[\mathrm{CO}_{2}(74 \%)\right.$ and $\left.\operatorname{Ar}(79 \%)\right]$ and we observed that $d_{b}$ varied about $14 \%$. These comparisons clearly show that both properties can influence the early stage of impact.

Regarding the role of compressibility, the model of Mandre et al. [13] predicts that the gas bubble diameter becomes smaller when $\epsilon^{-1}$ increases, but our experiment demonstrates the opposite. For example, we found that for $\mathrm{He}\left(95 \%, \epsilon^{-1}=89\right)$ a diameter of $210 \mu \mathrm{m}$ is formed, while for $\mathrm{CO}_{2}\left(74 \%, \epsilon^{-1}=98\right)$ the diameter is $330 \mu \mathrm{m}$. The reason for this difference probably lies in the theoretical approach of their model, which assumes either an isothermal or adiabatic gas state. Hicks and Purvis [14] show with a full analysis of this gas, including energy conservation equations, that the gas bubble diameter is independent of the compressibility. This is the result of an interaction between the gas density, temperature, and interface which leads to greater lateral spreading of the gas than that predicted by Mandre et al. [13]; therefore, the decrease in size is compensated due to energy conservation. Since the gas bubble diameter is independent of the compressibility, the diameter can be predicted using the previous developed incompressible theory of Hicks and Purvis [23]. It concludes that

$$
d_{b}=K\left(\frac{4 \mu_{g}}{\rho_{l} U}\right)^{1 / 3}(D / 2)^{2 / 3}
$$


with $K=3.8$ as calculated in Ref. [14]. This formula agrees with the experimental data obtained by Li and Thoroddsen [24], where they investigated the droplet impact using only air as a surrounding gas. As shown in Fig. 3, this incompressible model predicts $d_{b}$ accurately only for our low-density gases, even at high Weber and Reynolds numbers. By increasing the gas density, the model predicts almost the same diameter while our measurements clearly demonstrated an increase. We attribute this discrepancy to the absence of gas density in Eq. 1, which increases in relation to $\rho_{g}$. The slight deformation of the primary droplet observed during experiments with $\mathrm{SF}_{6}$ probably led to more entrapment than expected for a perfectly spherical droplet [24]. Considering this effect in Eq. (1), the model predicts a diameter of $\approx 250 \mu \mathrm{m}$ and, consequently, droplet deformation caused by substrate movement is insufficient to explain the discrepancy observed.

\section{B. The size and velocity of the secondary droplets}

Although a small amount of gas was entrapped regardless of which gas was used, splashing was not always observed when using helium or $\mathrm{NH}_{3}(77 \%)$. Xu et al. [8] studied the droplet impact at reduced pressure and concluded that the stress on the expanding lamella destabilizes the liquid and leads to its breakup. They estimated this stress as $\Sigma=0.5 \rho^{*} \mathrm{Ma}^{-1} \mathrm{WeRe}^{-1 / 2}$ with $\mathrm{Ma}$ as the Mach number and $\rho^{*}=\rho_{g} / \rho_{l}$. When $\Sigma>0.45$, splashing is expected. The use of this simple equation with our configurations indicates that for $\mathrm{He}(95 \%)$ no splash should be expected. For $\mathrm{NH}_{3}(77 \%)$ a stress of $\Sigma=0.46$ is estimated; this specific case is at the splash limit. In fact, we observed splashing in some of the images, demonstrating that a small change in the impact condition would provoke splashing. On the contrary, for $\mathrm{He}(70 \%)$ a stress of $\Sigma=0.61$ is estimated, clearly suggesting splash. However, our measurements show similar behavior as for $\mathrm{NH}_{3}(77 \%)$, where splashing was only observed in some images.

In a more detailed theoretical investigation, Riboux and Gordillo [7] considered an inviscid and incompressible flow and concluded that splashing is attributed to a lift force experienced by spreading lamella. Their theoretical analysis shows that this lift force results from the lubrication force induced by the gas beneath the lamella and the suction force induced at the top of it. If the ratio of this lift force to surface tension $\beta=\sqrt{F_{L} /(2 \sigma)}$ is larger than 0.14 , then splashing is expected.

Similar to the stress analysis of $\mathrm{Xu}$ et al. [8], the splashing parameter $\beta$ predicts only the absence of splashing for $\mathrm{He}(95 \%)$. However, this theory indicates that the cases with $\mathrm{He}(70 \%)$ and $\mathrm{NH}_{3}(77 \%)$ are very close to the splashing threshold, with $\beta=0.16$ and 0.19 , respectively. The estimation of splashing proposed by Riboux and Gordillo [7] is therefore in better agreement with our observations. To finalize this section on what evokes splashing, we concluded based on the existing theoretical models, the previous observations made by Liu et al. [25], and our experiments that the entrapment of gas is not the mechanism responsible for triggering splashing. Hence, to investigate this physical process, we focus the following discussion on the secondary droplets that are generated.

Figure 4(a) shows the size distribution of the secondary droplets obtained by using different gases. This diagram demonstrates that the secondary droplet size is independent of the surrounding gas, even though the number of droplets detected increases with the gas density. Similar to our observations, Lakta et al. [26] qualitatively reported the ejection of more droplets with increased pressure. Note that the gas density is proportional to pressure. Thoroddsen et al. [6] suggest that the droplet size in early stages can be estimated because they are generated at the tip of fingerlike jets, which are formed at the rim of the spreading lamella. This assumption leads to $d_{s} \simeq 4 \sqrt{\mu_{l} t / \rho_{l}}$. This model neglects any other droplet formation mechanisms that occur at later stages, such as jet-merging and satellite droplets [27]. While the model predicts a maximal droplet size of $d_{s}=0.04 D$, our measurements revealed that their size never exceeded $d_{s}=0.02 D$. This difference can be attributed to an incorrect estimation of the rim dimensions at high impact velocities.

To calculate the diameter of the secondary droplets using the model presented by Riboux and Gordillo [7], it is necessary to first compute the state of the lamella at the ejection instant. This state can be characterized by the ejection time $\left(\tau_{e}\right)$, thickness $\left(h_{l}\right)$, and velocity $\left(u_{l}\right)$ of the lamella. These 

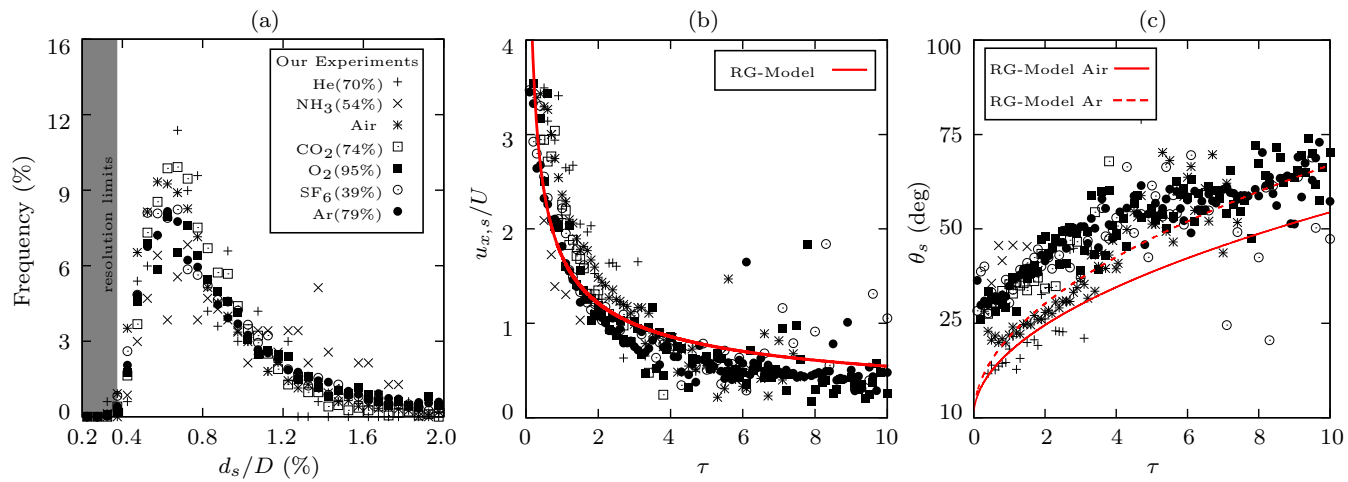

FIG. 4. Secondary ejected droplets. Diagram (a) shows the droplet size distribution, which is independent of the surrounding gas. Diagram (b) shows the horizontal velocity as a function of time, which is also independent of the gas. Diagram (c) shows the angle of the droplets relative to the surface as a function of time. The magnitudes of the standard deviations are $\sigma_{\tau}=0.1, \sigma_{u_{x, s}}=0.5 \mathrm{~m} / \mathrm{s}$, and $\sigma_{\theta_{s}}=7^{\circ}$.

are calculated as

$$
c_{1} \operatorname{Re}^{-1} \tau_{e}^{-1 / 2}+\operatorname{Re}^{-2} O h^{-2}=c \tau_{e}^{3 / 2}, \quad h_{l}=\frac{\sqrt{12} \tau_{e}^{3 / 2}}{\pi}, \quad \text { and } \quad u_{l}=\frac{1}{2} \sqrt{\frac{3}{\tau_{e}}},
$$

where $c_{1}=\sqrt{3} / 2, c=1.21, \operatorname{Re}=\rho U D /(2 \mu)$, and $O h=\mu / \sqrt{\rho D / 2 \sigma}$. The dimensional formulation of the ejection time, thickness, and velocity are obtained as $T_{e}=\tau_{e} D /(2 U), H_{l}=h_{l} D / 2$, and $U_{l}=u_{l} U$. In the case of a prompt splash, the size and velocity of the ejected droplets correspond to the thickness and velocity of the spreading rim (see Fig. 1). Thus, the viscous shear force at the rim cannot be neglected because this force simultaneously decreases the velocity of the rim from $u_{a}$ to $u_{a}^{+}$, while it increases the rim thickness from $h_{a}$ to $h_{a}^{+}$over time. Riboux and Gordillo considered this effect in Ref. [15] and derived the initial conditions of the evolution of the lamella, which read

$$
h_{a}^{+}=\frac{h_{a}}{\left(1-\sqrt{2} / \sqrt{\operatorname{Re} v_{a} h_{a}}\right)} \quad \text { and } \quad u_{a}^{+}=u_{a}\left(1-\sqrt{2} / \sqrt{\operatorname{Re} u_{a} h_{a}}\right),
$$

with $h_{a}=h_{l} / 3$ and $u_{a}=2 u_{l}$. Finally, the droplet size and velocity can be determined by $d_{\text {model }} \sim$ $R h_{a}^{+}$and $u_{\text {model }} \sim U u_{a}^{+}$. The theory reveals that the first secondary droplets are ejected at $T_{e} \simeq 1.7 \mu \mathrm{s}$ with $d_{\text {model }} \sim 3 \mu \mathrm{m}$ and $u_{x \text {, model }} \sim 80 \mathrm{~m} / \mathrm{s}$, which are similar to the values measured by Thoroddsen et al. [6]. However, to compare this model to our results, the elapsed time for the generation of secondary droplets must be known; otherwise, the model predicts a continuous increase in the droplet size over time. According to the experiments of Thoroddsen et al. [6], which are the closest to our configuration, the majority of the secondary droplets were generated in the first $100 \mu \mathrm{s}$ after impact, i.e., $\tau=0.2$. If this is also true in our experiments, then the mean diameter of the ejected secondary droplets predicted by the model is $\bar{d}_{\text {model }} \approx 25 \mu \mathrm{m}$ for all the studied cases; while we measured $\bar{d}_{s} \approx 27 \mu \mathrm{m}$.

Figure 4(b) shows the horizontal velocity of the droplets $u_{x, s}$ over the dimensionless time $\tau=$ $t U D^{-1}$. Similar to our previous conclusion, the horizontal velocity is almost independent of the gas. This evolution over time using Eqs. (2) and (3) is also predicted by theory. This reinforces the previous observation made by different authors, where $U \sim \tau^{-1 / 2}[6,7,28]$. Contrary to the droplet size and horizontal velocity, the vertical velocity of the droplets $u_{y, s}$ is strongly affected by the gas, as can be seen in the evolution of the angles in Fig. 4(c). The theory of Riboux and Gordillo allows calculation of the vertical velocity as $u_{y \text {, model }} \sim 10 U \sqrt{F_{L} /\left(\rho H_{l} U_{l}^{2}\right)}$ and shows agreement with the droplet angle measurements. Note that the droplet angle of Riboux and Gordillo is defined by the angle of the spreading lamella. However, the measurements presented here capture the droplets once 
they have detached from the lamella. After the detachment, the aerodynamic forces on the droplets, for example, drag or lift, may play an important role and should be considered. We assume that these aerodynamic forces are in fact responsible for the offset between our measurements and the prediction. Nevertheless, the tendency is well described by the model. In the numerical investigation performed by Guo et al. [11], the influence of the surrounding gas has also been attributed to the vertical deflection of the lamella as a result of the aerodynamic forces. We have demonstrated these effects here for the first time by measuring the secondary droplets ejected.

\section{The total ejected volume}

Although the characterization of the secondary droplets has revealed important information about splashing, it is still unknown how much volume of the primary droplet flies away during splashing. To quantify this, we calculated the total volume of the secondary droplets $V_{s}$ as follows. First, we determined the volume of each droplet $V_{i}=\pi d_{s, i}^{3} / 6$. Second, we estimated the position that each droplet would have at every point in time for the remainder of the splash. Third, we defined a fixed control surface at 1.1 times the maximal spreading diameter as shown in Fig. 2(c). Fourth, we performed a radial extrapolation of only the droplets that pass through the control surface. The extrapolated volume flux for each $\Delta \tau$ is defined as

$$
\Phi_{s}=2 \pi \sum_{i=1}^{N} \frac{\left(x_{i}-x_{\mathrm{imp}}\right)}{\psi_{i}} V_{i},
$$

where $x_{i}$ is the droplet position before crossing the control surface, $x_{\text {imp }}$ the impact center, and, $\psi_{i}$ the individual depth of field (DOF). Finally, the total volume is obtained after integrating $\Phi_{s}$ over time. Note that by taking the DOF of each droplet into account, we correct the detection probability bias, as explained in Sec. II, and increase the accuracy with which the total volume is calculated. The total number of droplets $N$ is estimated by counting the number of droplets which have been extrapolated. This method is based on the work of Faßmann et al. [29], except here the volume is only extrapolated from the droplets passing over a control surface, eliminating the necessity of weighting factors. We would like to emphasize that we only evaluated droplets larger than $15 \mu \mathrm{m}$, and according to our estimate using the theory of Riboux and Gordillo [7], the smallest droplets should be $3 \mu \mathrm{m}$ in diameter. Thus, a small amount of volume could not be measured; however, the contribution of these small droplets $\left(V_{3 \mu \mathrm{m}}=1.41 \times 10^{-17} \mathrm{~m}^{3}\right)$ to the total volume is insignificant in comparison with the volume of larger droplets $\left(V_{50 \mu \mathrm{m}}=6.54 \times 10^{-14} \mathrm{~m}^{3}\right)$. For that reason, we expect that our results would not be greatly affected by capturing all droplets smaller than $15 \mu \mathrm{m}$.

The images in Fig. 5 show the outcome of splashing when the properties of the surrounding gas change at constant ambient pressure. To determined which gas property affects these observations the most, we compare two gases with similar viscosities but significantly different densities, $\Delta \rho_{g} \approx$ $60 \%\left[\mathrm{He}(70 \%)\right.$ and air], and we observed that the total volume loss $V_{s} / V_{D}$ differed by approximately $99 \%$. By comparing another two gases with similar densities but different viscosities, $\Delta \mu_{g} \approx 30 \%$ $\left[\mathrm{CO}_{2}(74 \%)\right.$ and $\left.\operatorname{Ar}(79 \%)\right]$, we observed that $V_{s} / V_{D}$ varied by approximately $34 \%$. When we compared $\mathrm{He}(95 \%)$ with $\mathrm{He}(70 \%)$, the mean free path changes $\Delta \lambda_{g}=50 \%$ but $\Delta \mu_{g}<7 \%$, and we note that the total volume loss $V_{s} / V_{D}$ changed from 0 to $2 \%$, suggesting that the mean free path has almost no influence on the total volume, while considering that $\lambda_{g} \propto \rho_{g}^{-1}$. To summarize, we concluded that the most dominant gas property affecting the generation of secondary droplets is the density, followed by viscosity and mean free path. This conclusion is also valid for the total number of droplets ejected, since the droplet size distribution is independent of the gas property, therefore, $N \sim V_{s}$. As a result, the measurement of the total number of droplets ejected for a single impact results in the following amounts $N_{\mathrm{He}(70 \%)} \approx 100, N_{\mathrm{NH}_{3}(77 \%)} \approx 3500, N_{\mathrm{NH}_{3}(54 \%)} \approx 4200, N_{\mathrm{Air}} \approx 17000, N_{\mathrm{O}_{2}(95 \%)} \approx$ $24000, N_{\mathrm{CO}_{2}(74 \%)} \approx 28000, N_{\mathrm{CO}_{2}(41 \%)} \approx 23000, N_{\mathrm{Ar}(79 \%)} \approx 40000$ and $N_{\mathrm{SF}_{6}(39 \%)} \approx 35000$.

To make use of the theory of Riboux and Gordillo [7] to predict the total ejected volume, we analyzed the forces acting on the spreading lamella, from which the droplets are generated. According to the authors, the generation of secondary droplets is caused by a lift force on the 

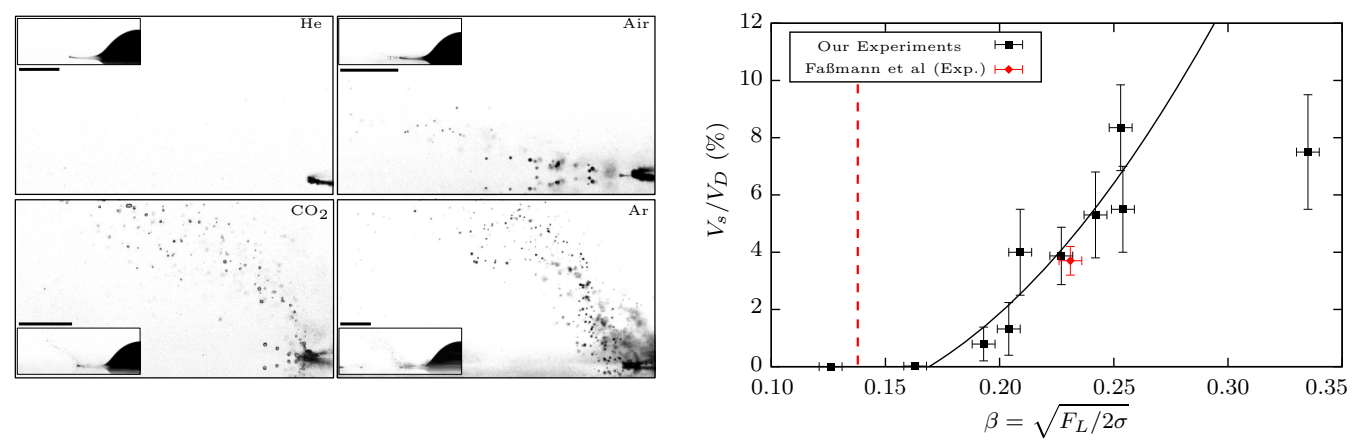

FIG. 5. Total volume ejected during splashing. The images demonstrate the role of the surrounding gas on the splashing outcome; scale bars $500 \mu \mathrm{m}$. The diagram shows the total ejected volume normalized to the primary droplet volume $V_{D}$ over the splashing parameter $\beta$. The solid line shows Eq. (6). The red dash line represents the splashing threshold $\beta=0.14$.

spreading lamella, which results from combining the lubrication force exerted by the gas beneath the lamella and the aerodynamic suction force exerted above the lamella. The formulation of the lift force is

$$
F_{L}=K_{\mathrm{lub}} \mu_{g} U_{l}+K_{u} \rho_{g} U_{l}^{2} H_{l},
$$

where $K_{\text {lub }} \simeq-2\left[\ln \left(19.2 \lambda_{g} / H_{l}\right)-\ln \left(1+19.2 \lambda_{g} / H_{l}\right)\right]$ is obtained from the lubrication theory and $K_{u}=0.3$ is a numerically determined constant. This expression of $F_{L}$ involves all of the previously mentioned gas properties. Moreover, this theory establishes that by increasing the vertical velocity of the lamella, due to the lift force, there is a point where this velocity is equal to the capillary retraction velocity; above this limit the lamella gradually starts to fragment into secondary droplets. The balance between these velocities leads to the previously mentioned splashing parameter $\beta=$ $\sqrt{F_{L} /(2 \sigma)}$; as a consequence, we expect that this ratio is directly proportional to the quantity of ejected droplets. To verify this expectation, in Fig. 5 we plot the total volume ejected as a function of $\beta$. The diagram demonstrates that the total ejected volume increases with the splashing parameter $\beta$. Based on our measurements, the available experimental data, and the theory of Riboux and Gordillo we provide an expression for the total volume ejected,

$$
\frac{V_{s}}{V_{D}}=C_{1} \beta^{2}-C_{2} \beta+C_{3}
$$

where $C_{1}=3.8, C_{2}=0.8$, and $C_{3}=0.026$ are constants determined empirically. $\operatorname{SF}_{6}(\beta=0.33)$ is the outlier in our data. In this case, as we mentioned previously, the primary droplet slightly deformed to an oblate before impact due to the high stagnation pressure at the impact surface caused by the flywheel rotation. This could also decelerate the droplet leading to a lower impact velocity, which would consequently result in a lower $\beta$ value. Measurements at increased ambient pressure and at similar Weber and Reynolds numbers would help corroborate this estimate. Such experiments are challenging because even higher impact velocities would be necessary to compensate the deceleration of the droplets before impact, which would again lead to larger deformation. Nevertheless, the evaluation of more than 1000 individual impacts in this study provide a solid dataset for statistical determination of the impact outcome, which agrees with the existing theory. Our experimental investigation elucidates quantitatively and qualitatively the role of the surrounding gas in the outcome of splashing.

\section{CONCLUSIONS}

This experimental study shows that at the early stage of impact, the properties of the gas affect the morphology and size of the entrapped gas bubble. The first contact with the surface commonly 
leaves a ring of microbubbles with single or multiple larger bubbles in the center, as observed by Thoroddsen et al. [22]. The use of helium inhibited the formation of the ring of microbubbles; instead a single bubble was observed. In that particular case, the mean free path of the gas was larger than the estimated distance between the bottom of the droplet and the surface at which the droplet starts to deform. In accordance with Mandre and Brenner [21], we attribute gas entrapment to gas compression beneath the droplet, which again leads to entrapment.

The initial size of the entrapped gas was analyzed by measuring the diameter of the ring with microbubbles. The measurements show an increase in this diameter with increasing density or viscosity of the gas. We compared our results with the theoretical models developed by Mandre et al. [13] and Hicks and Purvis [14], where compressibility effects have been taken into account. The models predict either a decrease or a constant size with increasing density, while our measurements demonstrate the opposite. However, the model proposed by Hicks and Purvis [23] agrees well with our observations using low density gases $\left(\rho_{g}<1.25 \mathrm{~kg} / \mathrm{m}^{3}\right)$. We observed the entrapment of gas in all experiments conducted, even though no secondary droplets were ejected in some cases. This let us conclude that droplet deformation at the early stage of impact is not the mechanism responsible for splashing, as has been hypothesized by different authors [7,25,30].

The surrounding gas mainly affects the angle and the number of secondary droplets ejected, while the droplet size distribution and horizontal velocity are independent of the gas properties. By combining our experimental findings with a profound analysis of the theory proposed by Riboux and Gordillo [7], we substantiate the important role of the aerodynamic lift force exerted on the lamella during splashing. Furthermore, this lift force is influenced primarily by the density, followed by the viscosity, and finally by the mean free path of the surrounding gas. The size and velocity average of the secondary droplets can be accurately calculated using the extended model of Riboux and Gordillo [15]. However, the number of droplets which are ejected with a certain size, specifically the frequency distribution, remains unsolved by the models.

Our estimation of the total ejected volume also indicates a strong dependence on the surrounding gas, which is analogous to our measurements of the number of ejected droplets. As mentioned above, the droplet size distribution is found to be independent of the gas used; this allows us to use a simplified model to calculate the total volume ejected. Based on our findings, we use the theory of Riboux and Gordillo [7] to provide the first expression for the total volume of impacts on smooth dry surfaces.

\section{ACKNOWLEDGMENTS}

We thank G. Riboux and J. M. Gordillo for their valuable comments that helped us to use their model, J. C. Londoño for conducting part of the experiments, and the Deutsche Forschungsgemeinschaft DFG for the financial support (Grant No. BA 4953/3).

[1] C. Josserand and S. Thoroddsen, Drop impact on a solid surface, Annu. Rev. Fluid Mech. 48, 365 (2016).

[2] C. Günther, J. Bröder, and F. Joos, Influence of pressure on droplet splashing behavior inside gas turbine compressors during wet compression, in Proceedings of the 17th International Symposium on Transport Phenomena and Dynamics of Rotating Machinery (ISROMAC-17), Forum 36, 16-21 December, Maui, Hawaii (2017).

[3] W. Wright and M. Potapczuk, Semi-empirical modelling of SLD physics, in Proceedings of the 42nd AIAA Aerospace Sciences Meeting and Exhibit, 5-8 January, Reno, Nevada, paper 20040412 (AIAA, Reston, VA, 2004).

[4] P. Trontin and P. Villedieu, Revisited model for supercooled large droplet impact onto a solid surface, J. Aircr. 54, 1189 (2016).

[5] L. Xu, Liquid drop splashing on smooth, rough, and textured surfaces, Phys. Rev. E 75, 056316 (2007).

[6] S. T. Thoroddsen, K. Takehara, and T. G. Etoh, Micro-splashing by drop impacts, J. Fluid Mech. 706, 560 (2012). 
[7] G. Riboux and J. M. Gordillo, Experiments of Drops Impacting a Smooth Solid Surface: A Model of the Critical Impact Speed for Drop Splashing, Phys. Rev. Lett. 113, 024507 (2014).

[8] L. Xu, W. W. Zhang, and S. R. Nagel, Drop Splashing on a Dry Smooth Surface, Phys. Rev. Lett. 94, 184505 (2005).

[9] S. T. Thoroddsen, T. G. Etoh, and K. Takehara, Air entrapment under an impacting drop, J. Fluid Mech. 478, 125 (2003).

[10] C. S. Stevens, A. Latka, and S. R. Nagel, Comparison of splashing in high-and low-viscosity liquids, Phys. Rev. E 89, 063006 (2014).

[11] Y. Guo, Y. Lian, and M. Sussman, Investigation of drop impact on dry and wet surfaces with consideration of surrounding air, Phys. Fluids 28, 073303 (2016).

[12] Z. Jian, C. Josserand, S. Popinet, P. Ray, and S. Zaleski, Two mechanisms of droplet splashing on a solid substrate, J. Fluid Mech. 835, 1065 (2018).

[13] S. Mandre, M. Mani, and M. P. Brenner, Precursors to Splashing of Liquid Droplets on a Solid Surface, Phys. Rev. Lett. 102, 134502 (2009).

[14] P. D. Hicks and R. Purvis, Liquid-solid impacts with compressible gas cushioning, J. Fluid Mech. 735, 120 (2013).

[15] G. Riboux and J. M. Gordillo, The diameters and velocities of the droplets ejected after splashing, J. Fluid Mech. 772, 630 (2015).

[16] C. R. Wilke, A viscosity equation for gas mixtures, J. Chem. Phys. 18, 517 (1950).

[17] S. G. Jenning, The mean free path in air, J. Aerosol Sci. 19, 159 (1988).

[18] K. S. Kim and S. S. Kim, Drop sizing and depth-of-field correction in TV imaging, Atom. Sprays 4, 1 (1994).

[19] F. T. Smith, L. Li, and G. X. Wu, Air cushioning with a lubrication/inviscid balance, J. Fluid Mech. 482, 291 (2003).

[20] S. Shen, G. Chen, R. M. Crone, and M. Anaya-Dufresne, A kinetic-theory based first-order slip boundary condition for gas flow, Phys. Fluids 19, 086101 (2007).

[21] S. Mandre and M. P. Brenner, The mechanism of a splash on a dry solid surface, J. Fluid Mech. 690, 148 (2012).

[22] S. T. Thoroddsen, T. G. Etoh, K. Takehara, N. Ootsuka, and Y. Hatsuki, The air bubble entrapped under a drop impacting on a solid surface, J. Fluid Mech. 545, 203 (2005).

[23] P. D. Hicks and R. Purvis, Air cushioning and bubble entrapment in three-dimensional droplet impacts, J. Fluid Mech. 649, 135 (2010).

[24] E. Q. Li and S. T. Thoroddsen, Time-resolved imaging of a compressible air disc under a drop impacting on a solid surface, J. Fluid Mech. 780, 636 (2015).

[25] Y. Liu, P. Tan, and L. Xu, Kelvin-Helmholtz instability in an ultrathin air film causes drop splashing on smooth surfaces, Proc. Natl. Acad. Sci. USA 112, 3280 (2015).

[26] A. Latka, A. Strandburg-Peshkin, M. M. Driscoll, C. S. Stevens, and S. R. Nagel, Creation of Prompt and Thin-Sheet Splashing by Varying Surface Roughness or Increasing Air Pressure, Phys. Rev. Lett. 109, 054501 (2012).

[27] Y. Wang and L. Bourouiba, Unsteady sheet fragmentation: Droplet sizes and speeds, J. Fluid Mech. 848, 946 (2018).

[28] D. A. Burzynski and S. E. Bansmer, High speed visualization of droplets impacting with a dry surface at high weber numbers, in New Results in Numerical and Experimental Fluid Mechanics XI, edited by A. Dillmann et al., Notes on Numerical Fluid Mechanics and Multidisciplinary Design (Springer, Cham, 2018), Vol. 136, pp. 511-521.

[29] B. W. Faßmann, S. E. Bansmer, T. J. Möller, R. Radespiel, and M. Hartmann, High velocity impingement of single droplets on a dry smooth surface, Exp. Fluids 54, 1516 (2013).

[30] M. M. Driscoll and S. R. Nagel, Ultrafast Interference Imaging of Air in Splashing Dynamics, Phys. Rev. Lett. 107, 154502 (2011). 